\title{
RNA interference of long-chain acyl-CoA synthetase 6 suppresses the neurite outgrowth of mouse neuroblastoma NB41A3 cells
}

\author{
HI CHUL KIM ${ }^{1,4^{*}}$, SAE-WON LEE ${ }^{1 *}$, YONG-YEON CHO ${ }^{1,5}$, JEONG MOOK LIM ${ }^{2}$, \\ ZAE YOUNG RYOO ${ }^{3}$ and EUN JU LEE ${ }^{1}$ \\ ${ }^{1}$ Clinical Research Institutes, Seoul National University Hospital, Seoul 110-744; ${ }^{2}$ Gamete and Stem Cell Biotechnology \\ Department of Agricultural Biotechnology, Seoul National University, Seoul 151-742; ${ }^{3}$ School of Life Science \\ and Biotechnology, Kyungpook National University, Daegu 702-701, Korea
}

Received February 6, 2009; Accepted May 25, 2009

DOI: 10.3892/mmr_00000155

\begin{abstract}
Lipogenesis is crucial during neuronal development. Abnormal lipid metabolism causes neurological disorders such as Refsum disease and contributes to tumor formation. Longchain acyl-CoA synthetase (Acsl) ligates coenzyme A to fatty acids, thereby activating the lipid metabolism pathway. Here, we designed a specific small interference RNA (siRNA) against mouse Acs16, pU6-487i and pU6-586i, and investigated the function of Acsl6 in neuron differentiation. Expression of mAcsl6 mRNA and protein was markedly decreased by pU6487i and pU6-586i in NB41A3 mouse neuroblastoma cells. We established two stable cell lines, NB41A3-487 and NB41 A3-586, which expressed mAcsl6 siRNA. Knockdown of the mAcsl6 gene inhibited the proliferation of NB41A3 cells; in NB41A3-586 cells neurite outgrowth was suppressed, while in NB41 A3-487 cells it was almost absent. In addition, pU6-487i was more effective than pU6-586i in the reduction of cell proliferation and neurite outgrowth. The decline noted in the growth curves as well as the neurite outgrowth resulting from mAcsl6 knockdown indicate that the mAcsl6 gene plays a pivotal role in neuron development.
\end{abstract}

\section{Introduction}

Fatty acids (FAs) are used as energy sources (1). In skeletal and cardiac muscles, FAs are required for the enormous amounts

Correspondence to: Dr Eun Ju Lee, Clinical Research Institutes, Seoul National University Hospital, Seoul 110-744, Korea

E-mail: leeunju@snu.ac.kr

Present addresses: ${ }^{4}$ Institute Pasteur Korea, Seoul, Korea; ${ }^{5}$ The Hormel Institute, University of Minnesota, Austin, MN, USA

${ }^{*}$ Contributed equally

Key words: mAcs16, neurite outgrowth, lipid metabolism, neuron development, small interference RNA, NB41A3 neuroblastoma cells of energy expended in myosin movement. In particular, lipogenesis is important in normal development and disease. Neurons require substantial amounts of FAs for phospholipid synthesis in order to produce additional plasma membranes for generating extensive neurites during neuronal development $(1,2)$. Long polyunsaturated fatty acids (PUFAs), which consist mainly of docosahexaenoic acid (DHA) and arachidonic acid (AA), are particularly enriched in the membrane phospholipids of neural tissue. In neurons, the synapses have the highest concentration of long PUFAs, particularly DHA and AA $(3,4)$. Abnormal lipid metabolism contributes to neurological disorders such as Refsum disease, an autosomal recessive lipid metabolic disorder (5). In addition, the overexpression of lipogenic enzymes is involved in tumor formation (6). Lipid metabolism is low in non-malignant tumors, whereas in many malignant tumors, including breast and prostate cancer, it is high (6).

FAs are activated by coenzyme A (CoA), which is ligated to the FAs by long-chain acyl-CoA synthetase (Acsl). This addition increases the solubility of the FAs, preparing them for use in one of the many lipid metabolism pathways (7). To date, five rodent Acsls have been identified and show wide tissue distribution. Acsl1 and 5 are the predominant isoforms in the liver and adipocytes, Acs14 predominates in adrenal tissue and other steroidogenic organs, and Acsl5 is expressed in the intestine (8-13). Acsl3 and 6 (originally Acsl2) are mainly expressed in the brain and in neural cell lines. However, during brain development only Acsl6 levels are elevated $(2,5,14,15)$. It has been reported that rat Acsl6 participates in neuronal cell proliferation and differentiation (15). Nonetheless, the role of the Acsl6 gene in neurite outgrowth is unclear.

We previously reported on a novel clone for a mouse homologue of the human Acsl6 gene, mouse Acs16 (mAcs16), describing four variants (mAcs16_v1, 2, 3 and 4) and three protein isoforms (mAcsl6a and $\mathrm{c} ; 78 \mathrm{kDa}$ and mAcs16b; $81 \mathrm{kDa})(16)$. In this study, we designed specific mAcsl6 siRNA which were able to knockdown four variants of mAcs16. We found that the designed mAcsl6 siRNA specifically suppressed mAcsl6 expression. Knockdown of mAcsl6 inhibited cell proliferation and neurite outgrowth in NB41A3 neuroblastoma cells, suggesting the importance of Acsl6 in neuron differentiation. 
Table I. Oligonucleotide sequences of the mAcsl6 siRNA inserts.

\begin{tabular}{|c|c|c|}
\hline & Sequence & Targeting \\
\hline $487 \mathrm{i}$ & $\begin{array}{l}\text { 5'-tttgGAAGCCAGAGCAACCTTACttcaagagaGTAAGGTTGCTCTGGCTTCttttt-3' } \\
\text { 3'-CTTCGGTCTCGTTGGAATGaagttctctCATTCCAACGAGACCGAAGaaaagatc-5' }\end{array}$ & 487-505 \\
\hline $586 \mathrm{i}$ & $\begin{array}{l}\text { 5'-tttgCACAGAGCAGTTCGTTGGTttcaagagaACCAACGAACTGCTCTGTGttttt-3' } \\
\text { 3'-GTGTCTCGTCAAGCAACCAaagttctetTGGTTGCTTGACGAGACACaaaagatc-5' }\end{array}$ & $586-604$ \\
\hline
\end{tabular}

Lowercase letters represent the overhang tail sequence; lowercase letters in bold, the loop structure sequence.

\section{Materials and methods}

Construction of the mAcsl6 RNAi vector. To generate the mouse Acsl6 (mAscl6) RNAi vector, two unique 19-nucleotide stretches within the mAcsl6 coding region at 487 bp (487i) and $586 \mathrm{bp}$ (586i) from the transcription start site were selected (GenBank accession no. AY786363). These regions are conserved in four mAcsl6 variants (16). The mAscl6 siRNA oligonucleotides were designed as sense strands of the 19-nucleotide sequences of $487 \mathrm{i}$ or $586 \mathrm{i}$, respectively, followed by a short spacer (5'-TTCAAGAGA-3'), along with the reverse complement of the sense strand and five thymidine residues as the U6 promoter transcriptional stop signal (Table I). The oligonucleotides were annealed, cloned into the $B b s I$ and $X b a \mathrm{I}$ sites of the mU6pro vector (obtained from the Turner Laboratory, University of Michigan; http:// sitemaker.umich.edu/dlturner.vectors) and named pU6mAcsl6RNAi (487i) and pU6-mAcsl6RNAi (586i). In order to introduce a selection marker into pU6-mAcsl6RNAi, a neomycin-cassette was amplified from pcDNA3.1 (Invitrogen, Carlsbad, CA, USA) using the primers SnaBI (5'-TACGTA ATTCTGTGGAATGTGTGTCAG-3') and NotI (5'-GTGGCC ATGATTACGCCAAGCTCT-3'). The amplified neomycincassette was incorporated into the pCR2.1-TOPO vector (Invitrogen) and susequently subcloned into the SnaBI/NotI site of pU6-mAcsl6RNAi (487i) or pU6-mAcsl6RNAi (586i). The final clones were verified by DNA sequencing and named pU6-487i or pU6-586i, respectively.

Cell culture and establishment of stable cell lines. The mouse neuroblastoma NB41A3 cell line was maintained at $37^{\circ} \mathrm{C}$ in a $5 \% \mathrm{CO}_{2}$ atmosphere in RPMI-1640 medium (Invitrogen) supplemented with $1 \%$ penicillin/streptomycin and $10 \%$ fetal bovine serum (HyClone, Logan, UT, USA). To establish stable cell lines, the NB41A3 cells were seeded at $1 \times 10^{4}$ cells/well in 6-well plates and cultured overnight. Cells reached $40 \%$ confluence prior to tranfection. pU6-487i or pU6-586i were transfected using Fugene 6 Transfection Reagent (Roche Diagnostics, Indianapolis, IN, USA) according to the manufacturer's protocols. Transfected cells were treated with G418 $(700 \mu \mathrm{g} / \mathrm{ml}$ ) (Promega, Madison, WI, USA) for 1 week. Each of the 10 clonal lines with pU6-487i or pU6-586i was expanded in a 35-mm well dish. The inhibitory effect of mAcsl6 RNAi was confirmed by reverse transcriptase-polymerase chain reaction (RT-PCR) analysis. The final stable transfectants were named NB41A3-487 or NB41 A3-586.
Neurite outgrowth analysis. Cells $\left(5 \times 10^{3}\right)$ were seeded onto plates coated with $35 \mathrm{~mm}$ collagen IV (Trevigen, Gaithersburg, MD, USA), and the neurite outgrowth assay was performed. After incubation for $24 \mathrm{~h}$, the medium was replaced with differentiation medium [0.25\% FBS, $1.5 \mu \mathrm{M}$ BSA (Sigma Chemical Co., St. Louis, MO, USA), $75 \mathrm{ng} / \mathrm{ml}$ nerve growth factor (Sigma) and RPMI-1640] and the cells were allowed to differentiate for 7 days. Neurite outgrowth was captured using a Leica DM IRB inverted microscope. In order to distinguish the neurites of each cell, the phase contrast image was converted to grayscale and the cell body was removed using the eraser function in Adobe Photoshop software (Adobe Systems Inc., San Jose, CA, USA). Quantification of the neuritis area was performed using TINA 2.0 (RayTest).

WST-1 assay. Cells $\left(3 \times 10^{3}\right)$ were seeded into 96-well plates at a final volume of $100 \mu \mathrm{l} /$ well culture medium. The WST-1 colorimetric assay measures the metabolic activity of viable cells based on the cleavage of tetrazolium salt WST-1 to formazan by mitochondrial dehydrogenase in live cells. The cell proliferation detection reagent WST-1 (Roche) was added to the cells at various time points. Formazan production was measured in an ELISA reader at a wavelength of $440 \mathrm{~nm}$ and normalized to the control wells containing medium only.

Reverse transcription-PCR. Cells were harvested with Trizol reagent (Invitrogen), and total RNA was extracted. Reverse transcription was conducted with $1 \mu \mathrm{g}$ of the total RNA using a cDNA Synthesis Kit (Promega). The amplification of cDNA fragments was performed by first heating at $98^{\circ} \mathrm{C}$ for $1 \mathrm{~min}$, followed by 27 cycles of $98^{\circ} \mathrm{C}$ for $20 \mathrm{sec}, 62^{\circ} \mathrm{C}$ (mAcs14-6) or $65^{\circ} \mathrm{C}$ (mAcsl1 and 3) for $1 \mathrm{~min}$, and $72^{\circ} \mathrm{C}$ for $1 \mathrm{~min}$, followed by a final extension step at $72^{\circ} \mathrm{C}$ for $10 \mathrm{~min}$. $\beta$-actin was used as an internal control. The primer sequences were obtained from GenBank (accession nos. AY786363 for mAcs16, BC056644 for Acsl1, BC031529 for Acsl3, BC058663 for Acs14, BC031544 for Acs15 and NM007393 for $\beta$-actin) and are listed in Table II. The PCR products were analyzed by gel electrophoresis and DNA sequencing.

Western blot analysis. Membrane protein was prepared as described previously (16). Stable NB41A3-487 and NB41A3-586 cells were homogenized in $20 \mathrm{mM}$ ice-cold lysis buffer (Tris- $\mathrm{HCl}, \mathrm{pH} 8.0$, and $150 \mathrm{mM} \mathrm{NaCl}$ ) containing a protease inhibitor cocktail (Roche), and were subsequently centrifuged at 3,000 rpm for $10 \mathrm{~min}$. The supernatant was 
Table II. Primer sequences for reverse transcription-PCR.

\begin{tabular}{|c|c|c|c|}
\hline Primer & Direction & Sequence $\left(5^{\prime}-3^{\prime}\right)$ & Product size (bp) \\
\hline \multirow[t]{2}{*}{ mAcsl6 } & Forward & CAGCATCACCATGTCATCCTGA & 900 \\
\hline & Reverse & CTGTTGTACCACTTGTGAAACA & \\
\hline \multirow[t]{2}{*}{ mAcsl1 } & Forward & ACATTCGGCAGTACGTGCGC & 1,108 \\
\hline & Reverse & AGCCCAACAGCCATCGCTTC & \\
\hline \multirow[t]{2}{*}{$\mathrm{mAcs} 13$} & Forward & AGTGTGGATGGCTTGGCTTC & 1,087 \\
\hline & Reverse & AGCAGCCTTCGGACATTCC & \\
\hline \multirow[t]{2}{*}{$\mathrm{mAcs} 14$} & Forward & CGACTCACTAGCTGTCATAG & 1,627 \\
\hline & Reverse & CTGATCACATAGGACTGGTC & \\
\hline \multirow[t]{2}{*}{ mAcsl5 } & Forward & GCTGATCAACAGACCTCAGC & 970 \\
\hline & Reverse & TCATAGACCCTGTTAAGGAG & \\
\hline \multirow[t]{2}{*}{$\beta$-actin } & Forward & GACATGGAGAAGATCTGGCA & 430 \\
\hline & Reverse & ATCTCCTGCTCGAAGTCTAG & \\
\hline
\end{tabular}

Gene-specific primers sequences were obtained from GenBank.

ultracentrifuged at 230,000 x g for $90 \mathrm{~min}$, and the pellets were resuspended in ice-cold suspension buffer $(50 \mathrm{mM}$ Tris- $\mathrm{HCl}$, pH 8.0, and $150 \mathrm{mM} \mathrm{NaCl}$ ] using a Teflon-glass homogenizer. Each membrane protein $(100 \mu \mathrm{g})$ was separated by SDSPAGE, transferred onto a nitrocellulose membrane (Schleicher \& Schuell, Dassel, Germany) and hybridized with anti-mAcs16 antibody (diluted 1:500) produced in rabbit (16). The mAcsl6 proteins were visualized with horseradish peroxidase-conjugated anti-rabbit IgG sheep antibody (diluted 1:2,000) and enhanced using chemiluminescence detection reagents (ECL; Amersham Pharmacia Biotech, Buckinghamshire, UK).

\section{Results}

DNA vector-based mAscl6 RNAi suppress the expression of $m A c s l 6$ in NB41A3 neuroblastoma cells. The mAscl6 gene has four transcription variants that encode three different proteins (16). To suppress the expression of the four mAscl6 transcripts, two unique 19-nucleotide stretches within the mAcsl6 coding region, at $487 \mathrm{bp}$ (487i) and $586 \mathrm{bp}$ (586i) from the transcription start site, were selected (Table I). These regions are conserved in four mAcsl6 variants. Two DNA vector-based RNA interference systems were used with the small nuclear RNA promoter U6 (mU6pro vector). The mAcsl6 RNAi vector, which allows for the prediction of the secondary structure of the siRNA transcript of the mAcs16 gene, was constructed (Fig. 1A).

Expression of mAcs16 mRNA was markedly decreased in the NB41A3-487 and NB41A3-586 cells (Fig. 1B). Western blotting also showed a dramatic decrease in the 78- and $81-\mathrm{kDa}$ mAcs16 proteins in the NB41A3-487 and NB41A3-586 cells compared with the non-transfected NB41A3 cells or mU6pro mock vector-transfected cells. (Fig. 1C). mAscl6 was not expressed in the liver, which was used as a negative control (Fig. 1C).
mAcsl6 knockdown decreased neuroblastoma proliferation. During development and normal cellular proliferation, lipid synthesis is crucial (2). We investigated whether mAscl6 knockdown could affect the growth of NB41A3 cells (Fig. 2). The WST-1 assay was used to determine cell viability and proliferation. The growth curve of mU6pro-NB41A3 mock transfectants peaked at day $4(96 \mathrm{~h})$, and then gradually decreased. The growth curve patterns of NB41A3-487 and NB41A3-586 were similar, and their proliferation was clearly decreased. By day 4 (96 h), NB41A3-487 growth had decreased by $50 \%$ and that of NB41A3-586 by $20 \%$ compared to the mU6pro mock vector-transfected cells (Fig. 2). This suggests that mAcsl6 gene knockdown inhibits the proliferation of NB41A3 cells, and that pU6-487i is more effective than pU6-586i in the reduction of cell proliferation.

Knockdown of mAcsl6 inhibited neurite outgrowth. Lipid synthesis is required for the production of plasma membrane, which generates neurites (1). We examined whether mAscl6 affects the neurite outgrowth of NB41A3 neuroblastoma cells (Fig. 3). The cells were cultured on collagen-coated dishes, and differentiation was induced by the addition of nerve growth factor for 7 days. In the control and mock mU6protransfected cells, neurite outgrowth was detected on day 1, and long outgrowth on day 7 (Fig. 3A, B, E and F), while in NB41A3-586 cells neurite outgrowth was very slow and short (Fig. 3D and H). In NB41A3-487 cells, neurite outgrowth was not induced (Fig. 3C and G), suggesting that pU6-487i is more effective than pU6-586i for neurite outgrowth in the mAscl6 knockdown.

As neurite outgrowth is a multifaceted process, and since the mAcsl gene family consists of five genes, we investigated whether mAcsl gene family expression is affected by mAcsl6 knockdown in NB41A3-487 cells (Fig. 4). The mAcsl1, 3, 4 and 
A

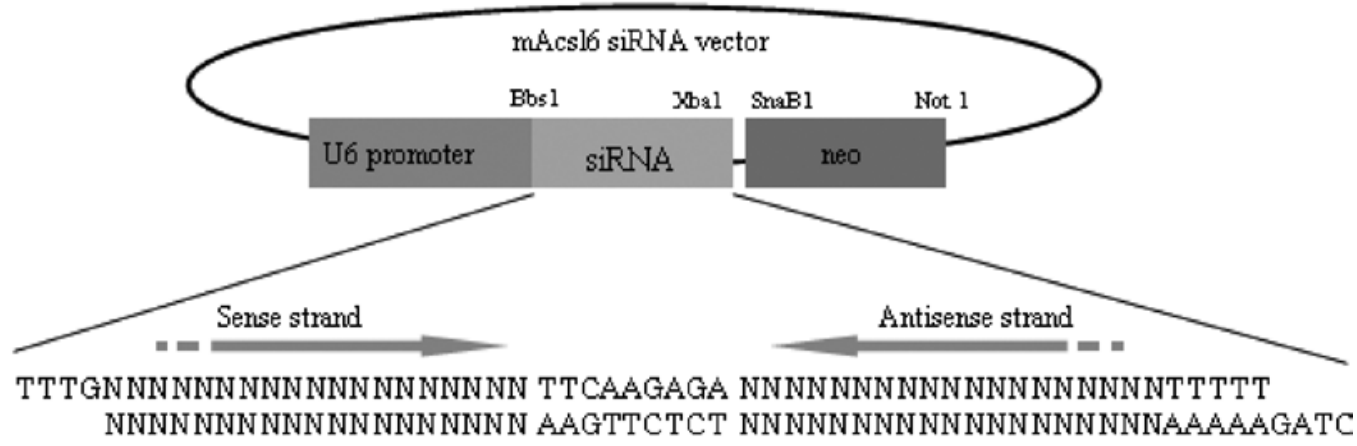

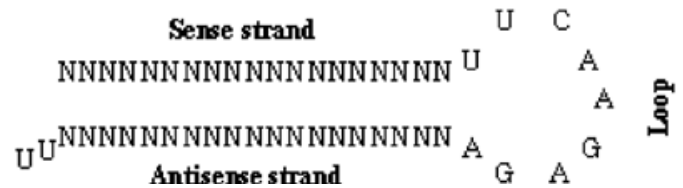

B
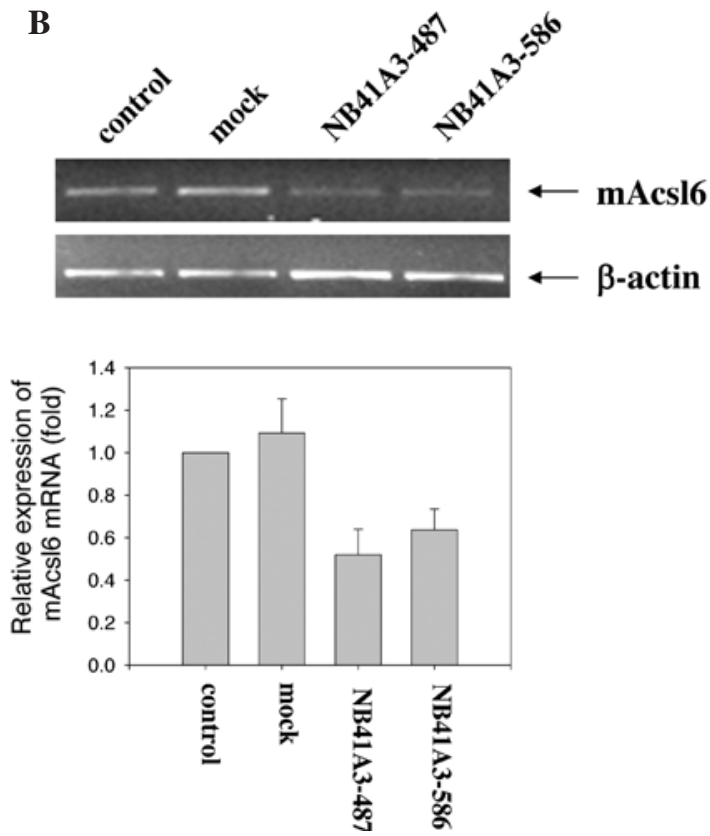

C
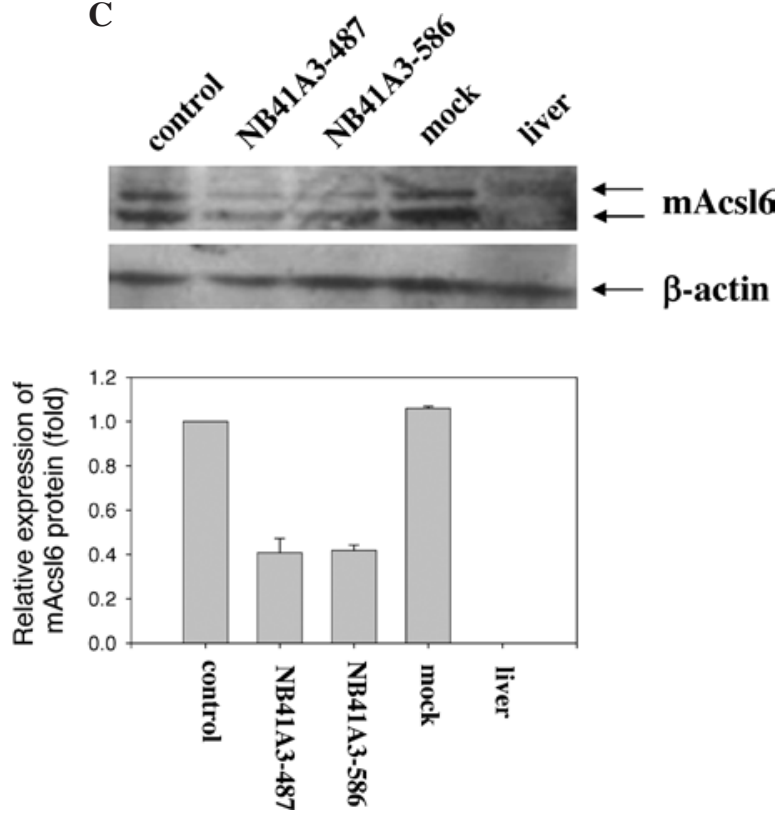

Figure 1. DNA vector-based mAcsl6 RNAi suppressed the expression of mAcsl6 in NB41A3 neuroblastoma cells. (A) Schematic representation of the mAcs16 siRNA vector. Two oligonucleotides containing sense and antisense 19-nucleotide sequences from the mAcsl6 gene and a transcription termination signal of five thymidines were inserted into pU6pro vector (top). The predicted secondary structure of the siRNA transcript was shown at the bottom. (B) RT-PCR assay of the inhibition of mAcs16 expression. The expression level of mAcs16 was significantly decreased in the two mAscl6 RNAi stable transfectants, NB41A3-487 and NB41A3-586, compared to the non-transfected NB41A3 cells (control) or mU6pro mock vector-transfected NB41A3 cells (mock). (C) Western blot analysis showed the reduced expression of mAcs16 protein in NB41A3-487 and NB41A3-586 cells. mAsc16 proteins were detected as 78- and 81-kDa. Liver protein was used as a negative control. The quantification of band intensity was conducted using TINA 2.0 and normalized to the intensity of the $\beta$-actin band.

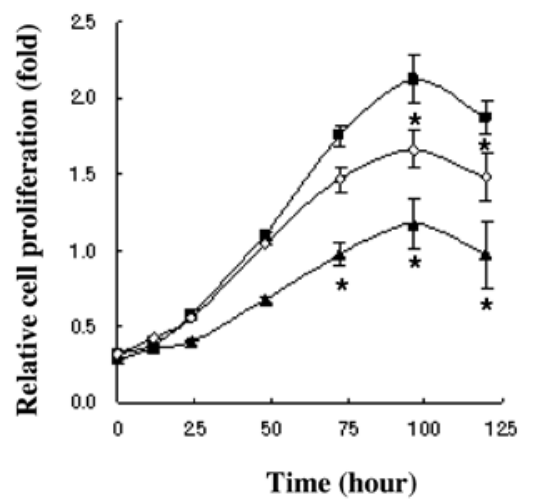

Time (hour)
Figure 2. Effect of mAcs16 RNAi on cell proliferation. NB41A3-487 and NB41A3-586 cells were cultured for 6 days. After $12 \mathrm{~h}$ of incubation in 96-well plates, the WST-1 assay was performed. mU6pro-NB41A3 mock cell proliferation peaked on day 4 ( $96 \mathrm{~h}$ ), and then gradually decreased (closed squares). Stable siRNA vector-transfected NB41A3 cells exhibited a similar pattern. In contrast, the proliferation of NB41A3-487 (closed triangles) and NB41A3-586 (open circles) decreased compared with the proliferation of the mU6pro mock cells (closed squares). A well that contained only culture medium served as the negative control. Data are presented as the means $\pm \mathrm{SD}$ $\left({ }^{*} \mathrm{p}<0.01\right)$ of three independent experiments. Each experiment was performed in triplicate. 

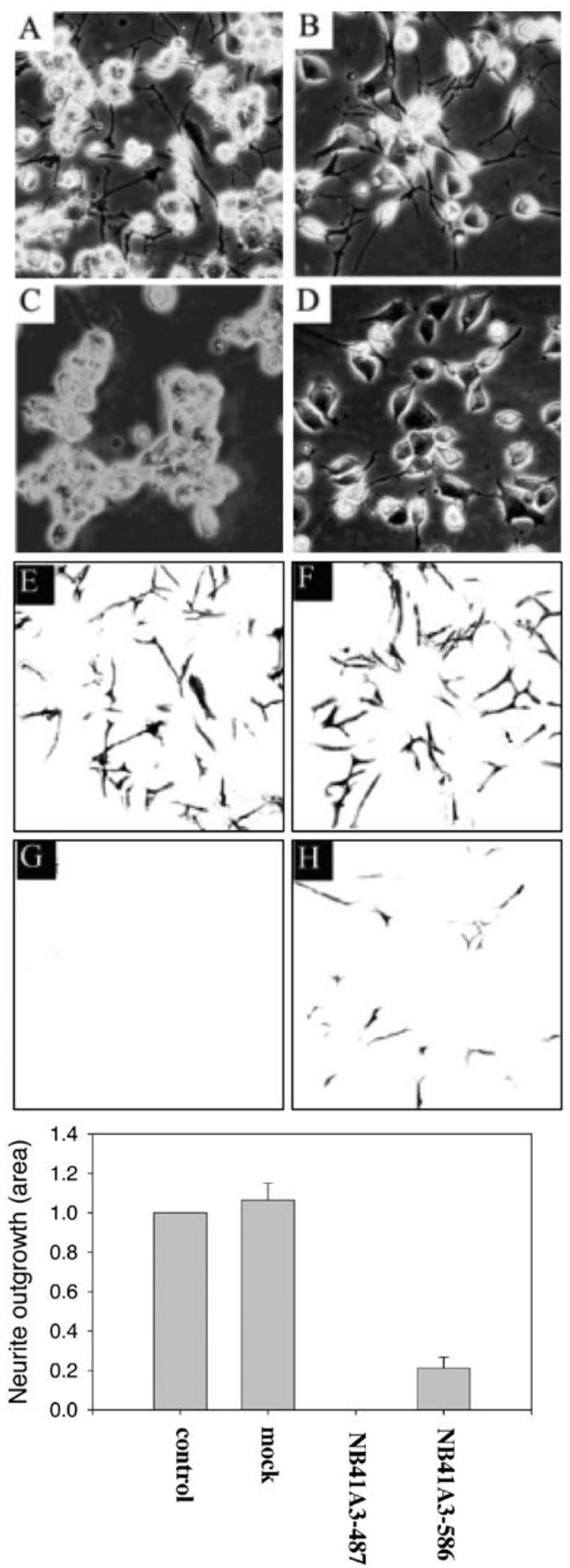

Figure 3. Inhibition of neurite outgrowth by mAcsl6 knockdown. Cells were cultured on 35-mm collagen-coated dishes and differentiated by the addition of nerve growth factor for 7 days. (A and E) Normal NB41A3 neuroblastoma, (B and F) mU6pro mock vector-transfected, (C and G) NB41A3-487 and (D and H) NB41A3-586 cells. The phase-contrast images were captured using a Leica DM IRB inverted microscope. Images were converted to grayscale and the cell body was removed using the eraser function in Adobe Photoshop, thus allowing clear visualization of the neurites of each cell (E-H). NB41A3-487 and NB41A3-586 cells exhibit short (D and $\mathrm{H})$ or no $(\mathrm{C}$ and $\mathrm{G})$ neurite growth. Quantification of neurite outgrowth (area) was performed and is shown at bottom. This experiment was performed independently in triplicate.

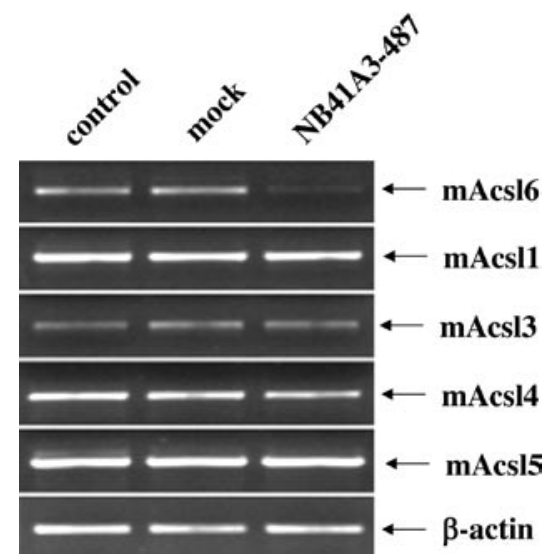

Figure 4. Expression of mAcsl genes in NB41A3-487 cells. Total RNA from NB41A3-487 cells was subjected to RT-PCR with specific primers for each member of the mAcsl gene family. Expression of the Acs11, 3, 4 and 5 genes was observed, and was not inhibited in NB41A3-487 cells. Only mAcs16 expression was decreased in NB41A3-487 cells. The $\beta$-actin gene was used as an internal control.

5 genes were not supressed by mAcs16 RNAi. This indicates that mAcsl6 siRNA is specific for mAcsl6 only, and that mAcs16 plays a key role in the neurite outgrowth of NB41A3 cells.

\section{Discussion}

During neurodevelopment, neurons increase phospholipid synthesis to generate the additional plasma membranes needed for neurite growth (1). FAs for neurite expansion are activated by Acsl, although it is unclear how Acsl contributes to neurodevelopment. The results of the proliferation and neurite outgrowth assays conducted in the present study elucidate the role of mAcsl6 in neuron development (Figs. 2 and 3). Inhibition of mAcsl6 markedly decreased neural proliferation and differentiation. In addition, two siRNA for mAcs16, 487i and 586i, showed definite differences in knockdown potency in the functional assay, but similar knockdown potency at the RNA and protein levels (Fig. 1). Although these two siRNAs for $\mathrm{mAcs} 16$ have identical G/C content, they exibited different knockdown potential. Thus, additional detailed and accurate criteria are needed to define the factors that determine siRNA functionality.

Reynolds et al listed criteria for the rational designing of potent siRNAs for functional gene knockdown studies (17). These criteria include low $\mathrm{G} / \mathrm{C}$ content, a bias towards low internal stability at the sense strand 3'-terminus, and a lack of inverted repeats and sense strand base preference (position 3, 10, 13 and 19) (17). Using these criteria, we can explain the differences observed between the pU6-487i and pU6-586i vectors in terms of neurite outgrowth and proliferation. The siRNA sequence of $487 \mathrm{i}$ matches the criteria to a greater extent than that of 586i, and might provide more effective and significant knockdown of the mAcsl6 gene.

The more functional NB41A3-487 cells were used to detect the expression of other mAcsl genes (Fig. 4). Assuming that neurite outgrowth is inhibited by the combined efforts of various mAcsl genes, and is not caused by the single effect of Acsl6 knockdown, we examined whether the expression of 
mAcsl genes might be regulated in mAcsl6 knockdown cells. The expression of the Acsl1, 3, 4 and 15 genes was not affected by mAcsl6 (Fig. 4), indicating that our mAcsl6 siRNA is very specific for mAcsl6 alone among the mAcsl gene family, and that mAcsl6 knockdown did not influence other mAcsl genes.

The inhibition of mAcsl6 is thought to affect various elements related to neurite outgrowth, such as acetylcholinesterase (AChE), the enzyme responsible for the rapid hydrolysis of acetylcholine in the central and peripheral nervous systems $(3,4,18,19)$. Expression of AChE was enhanced during neuronal differentiation (20). RT-PCR was performed to detect variations in AChE expression and found decreased AChE expression in NB41A3 cells blocked for mAcs16 expression (data not shown). Blast2 sequence analysis (http://www.ncbi.nlm.nih.gov/blast/bl2seq) showed no significant similarities between the transcripts of AChE and mAcsl6. This suggests that AChE gene knockdown is the result of the reduced expression of mAcsl6 and is not due to RNAi sequence homology. We propose that the immaturity of neurite outgrowth is due to a variety of factors associated with the neuronal system. Collectively, our results suggest that the mAcsl6 gene is crucial for neuronal proliferation and neurite outgrowth. Consequently, Acsl6 gene insufficiency should cause neuronal degeneration. A thorough understanding of the relationship between Acsl6 and neuron development may provide therapeutic solutions for neuronal disorders.

\section{References}

1. Innis SM: Essential fatty acids in growth and development. Prog Lipid Res 30: 39-103, 1991.

2. Marszalek JR, Kitidis C, Dararutana A and Lodish HF: Acyl-CoA synthetase 2 overexpression enhances fatty acid internalization and neurite outgrowth. J Biol Chem 279: 23882-23891, 2004.

3. Massoulie J, Pezzementi L, Bon S, Krejci E and Vallette FM: Molecular and cellular biology of cholinesterases. Prog Neurobiol 41: 31-91, 1993.

4. Taylor P and Radic Z: The cholinesterases: from genes to proteins. Annu Rev Pharmacol Toxicol 34: 281-320, 1994.

5. Kee HJ, Koh JT, Yang SY, Lee ZH, Baik YH and Kim KK: A novel murine long-chain acyl-CoA synthetase expressed in brain participates in neuronal cell proliferation. Biochem Biophys Res Commun 305: 925-933, 2003.
6. Brusselmans K, Schrijver DE, Verhoeven G and Swinnen JV: RNA interference-mediated silencing of the acetyl-CoA-carboxylasealpha gene induces growth inhibition and apoptosis of prostate cancer cells. Cancer Res 65: 6719-6725, 2005.

7. Black PN and DiRusso CC: Transmembrane movement of exogenous long-chain fatty acids: proteins, enzymes, and vectorial esterification. Microbiol Mol Biol Rev 67: 454-472, 2003.

8. Suzuki H, Kawarabayasi Y, Kondo J, Abe T, Nishikawa K, Kimura S, Hashimoto T and Yamamoto T: Structure and regulation of rat long-chain acyl-CoA synthetase. J Biol Chem 265: 8681-8685, 1990 .

9. Fujino T and Yamamoto T: Cloning and functional expression of a novel long-chain acyl-CoA synthetase expressed in brain. J Biol Chem 111: 197-203, 1992.

10. Fujino T, Kang MJ, Suzuki H, Iijima $H$ and Yamamoto T: Molecular characterization and expression of rat acyl-CoA synthetase 3. J Biol Chem 271: 16748-16752, 1996.

11. Kang MJ, Fujino T, Sasano H, Minekura H, Yabuki N, Nagura H, Iijima H and Yamamoto TT: A novel arachidonate-preferring acyl-CoA synthetase is present in steroidogenic cells of the rat adrenal, ovary, and testis. Proc Natl Acad Sci USA 94: 2880-2884, 1997.

12. Oikawa E, Iijima H, Suzuki T, Sasano H, Sato H, Kamataki A, Nagura H, Kang MJ, Fujino T, Suzuki H and Yamamoto TT: A novel acyl-CoA synthetase, ACS5, expressed in intestinal epithelial cells and proliferating preadipocytes. J Biol Chem 124: 679-685, 1998.

13. Malhotra KT, Malhotra K, Lubin BH and Kuypers FA: Identification and molecular chraracterization on acyl-CoA synthetase in human erythrocytes and erythroid precursors. J Biol Chem 344: 135-143, 1999.

14. Grand RJ: Acylation of viral and eukaryotic proteins. J Biol Chem 258: 625-638, 1989.

15. Setnik B and Nobrega JN: Long-chain acyl coenzymeA synthetase-2 mRNA: increased cerebral cortex expression in an animal model of depression. Prog Neuropsychopharmacol Biol Psychiatry 28: 577-582, 2004.

16. Lee EJ, Kim HC, Cho YY, Byun SJ, Lim JM, and Ryoo ZY: Alternative promotion of the mouse acyl-CoA synthetase 6 (mAcsl6) gene mediates the expression of multiple transcripts with 5'-end heterogeneity: genetic organization of mAcsl6 variants. Biochem Biophys Res Commun 327: 84-93, 2005.

17. Reynolds A, Leake D, Boese Q, Scaringe S, Marshall WS and Khvorova A: Rational siRNA design for RNA interference. Nat Biotechnol 22: 326-330, 2004.

18. Soreq H and Seidman S: Acetylcholinesterase - new roles for an old actor. Nat Rev Neurosci 2: 294-302, 2001.

19. Legay C: Why so many forms of Acetylcholin-esterase? Micro Res Tech 49: 56-72, 2000.

20. Deschenes FJ, Belanger G, Perrone BN and Jasmin BJ: Posttranscriptional regulation of acetylcholin-esterase mRNAs in nerve growth factor-treated PC12 cells by the RNA-binding protein HuD. J Biol Chem 265: 5710-5717, 2002. 